\title{
Fatigue life and backface strain predictions in adhesively bonded joints.
}

\author{
A. Graner Solana ${ }^{a}$, A.D. Crocombe ${ }^{a, \star}$ and I.A. Ashcroft ${ }^{b}$ \\ ${ }^{a}$ Faculty of Engineering and Physical Sciences (J5), University of Surrey, Guildford, \\ Surrey GU2 7XH, UK. \\ ${ }^{\mathrm{b}}$ Wolfson School of Mechanical and Manufacturing Engineering, Loughborough \\ University, Leicestershire LE11 3TU, UK \\ * Corresponding author. Tel: +441483689194 Fax: +441483 306039 E-mail \\ address:a.crocombe@surrey.ac.uk
}

\section{Abstract}

Fatigue is a very important factor in any adhesively bonded structure subject to service loads. Prediction of fatigue life using finite element analysis (FEA) techniques is very complicated due to the complex nature of fatigue damage. This paper presents experimental data obtained by testing single lap joints (SLJs) in constant amplitude fatigue at a range of load levels and associated fatigue damage modelling. Six strain gauges (SGs) placed along the overlap were used to monitor fatigue initiation and propagation within the adhesive layer. An elasto-plastic damage model was developed that was capable of predicting the experimentally observed backface strain patterns and fatigue life at different fatigue loads. It was implemented in the finite element code ABAQUS and used a user defined subroutine to calculate the damage, and the resultant degradation in adhesive Young's modulus and yield stress.

Keywords: epoxy/epoxides (A), aluminium and alloys (B), FE stress analysis (C), Fatigue (D), progressive damage modelling. 


\section{Introduction}

Fatigue loading is a common cause of failure in structures. Although much fatigue testing of adhesively bonded joints has been undertaken comparatively little work has been addressed at simulating the fatigue damage process. This paper addresses this important area. Several experimental and modelling approaches to detect and monitor damage initiation in adhesively bonded structures have been assessed. Recent work has included the use of the backface strain technique and video microscopy.

Quaresimin et al. [1] tested SLJs of varying overlap length in fatigue at different stress levels and conditions. The damage was monitored using video microscopy and a crack length of $0.3 \mathrm{~mm}$ was arbitrarily fixed as a threshold value for crack initiation. This damage was defined as a crack nucleation phase during which the adhesive whitened and crazed. This phase lasted between $20 \%$ and $70 \%$ of the fatigue life depending on the stress level and overlap..

On the other hand $\mathrm{Xu}$ et al. [2] observed the damage initiation as a process of microcrack formation. A series of microcracks appeared ahead of the tip and then merged into a major crack. This pattern was also seen by Apalak and Engin [3] in static joint testing. Dessureault and Spelt [4] also investigated this phenomenon in fatigue.. Crack initiation and propagation characteristics generally varied depending on the applied mode of loading, in mode I a similar microcrack formation process was observed. 
Ishii et al. [5] and Zeng and Sun [6] also used video microscopy to monitor fatigue damage in adhesive joints. Both used a different definition for crack initiation to Quaresimin et al. [1] Ishii et al. defined initiation as when the crack passed over the tip of the substrate corner contained in the fillet whilst Zeng and Sun used the first visible crack appearance.

A more complete method was applied by Cheuk et al. [7]. They tested double lap joints and used both video microscopy and SGs .They identified 2 different crack initiation scenarios; along the vertical interface on the end of the substrate and through the fillet.

The use of the backface strain technique in bonded joints was first assessed by Zhang et al. [8]. Their research with SLJ's concluded that crack initiation can be detected by the switch in the direction of the backface strain change.. However, Crocombe et al. [9] investigated the technique in more detail and showed that the backface strain response was highly dependent on its location. The research concluded that ideally the SG position should be inside the overlap because here it will produce the largest change in backface strain with damage.

Lefevre and Dillard [10] employed the backface strain technique to determine the number of cycles necessary for an interfacial crack to appear in an epoxy-aluminium epoxy wedge. The data from tests were used to develop a damage initiation map. Imanaka et al. [11] adopted a similar approach with adhesively bonded butt joints. The damage was 
evaluated as a change in the rigidity of the adhesive layer which was measured by two SGs connecting the upper and lower substrates across the adhesive layer.

The modelling approaches to fatigue also vary. Crocombe et al. [9] are amongst those who sought to correlate the fatigue performance against the stress field in the adhesive. Crocombe and Richardson [12] tested four different bonded joints configurations made with the same material, AV119 epoxy adhesive and steel. The effect of the mean load was investigated by performing fatigue tests at different load ratios. The results showed that the load-life data could be matched by using a Goodman type approach

Some authors have used the stress singularities. Lefevre and Dillard [10] defined crack initiation based on these stress singularities and developed a fatigue initiation map. Ishii et al. [5] also used the stress singularity to evaluate the fatigue strength of various lap joints. The fatigue strength was defined as an endurance limit, which depended on the apparent values of the stress intensity factor and of the stress singularity

Imanaka et al. [11] developed a continuum damage model, which was coupled with a kinetic law of damage evolution. The normalised apparent Young's modulus was very important in measuring the damage characteristics because it corresponded with the decrease of effective cross-sectional area due to the formation and growth of voids and microcracks. A damage variable $D$ was used to characterise this reduction in crosssectional area When the experimental and numerical results were compared there was a good match. 
Barrandon [13] explored the possibilities of a linear damage model based on the adhesive von Mises stress. The Young modulus decreased with the increasing damage He was then able to successfully simulate the measured experimental backface strain scenarios. Crocombe et al. [9] also developed and matched these backface strain scenarios. However, they relied on inserting cracks in the adhesive layer. A 3D model of an epoxyaluminium SLJ was developed. Crack growth was simulated by decoupling nodes on the interface between the adhesive and the substrate. This approach gave good results when fitting the experimental backface strain data. Finally, Quaresimin [1] proposed a damage model based on two phases, nucleation and propagation. In the first a generalized stress intensity factor was used, while in the second a derivative of the Paris law was applied.

This current work continues from the work reported earlier [14], where SLJs were tested and six SGs were used across the overlap width, to detect and monitor fatigue initiation Now the SGs have been placed at three different positions along the overlap, which allows a more thorough monitoring of damage progression. An elasto-plastic damage model has been developed, capable of predicting and matching backface strain patterns and fatigue life at different loads.

\section{The experimental tests}

The SLJ dimensions are shown in Table 1. The fatigue tests were conducted using an Instron 8511 fatigue machine in constant amplitude fatigue at $5 \mathrm{~Hz}$ with maximum 
fatigue loads of $50 \%, 40 \%$ and $30 \%$ of the static shear strength $\left(\tau_{\mathrm{S}}\right)$ and with a load ratio $R=0.1$. Static tests to failure were performed to assess the SLJs reliability in terms of strength. The static strength of the tested joints was very similar, $17 \mathrm{kN}$. The variation was less than $0.5 \mathrm{kN}$, thus reliability of the specimens was very good.

Six strain gauges were placed on the SLJs overlap. The gauges used were selftemperature compensated EA-13-060LZ-120 uniaxial SGs with a gauge length of $1.5 \mathrm{~mm}$. The SG positions with respect to the overlap can be seen in Figure 1. SG1-3 were placed at end A and SG4-6 at end B. SG2 and 5 were placed 1mm inside the overlap; SG3 and 6 were placed $3 \mathrm{~mm}$ inside the overlap; SG1 and 4 were placed $5 \mathrm{~mm}$ inside the overlap (Figure 1). The change in voltage was amplified and recorded using in-house datalogging software measuring maximum and minimum voltage values, and snap shots of the complete voltage change of all 6 SGs. The data were then converted into strain.

A total of 6 specimens were tested in fatigue and the load - life data are shown in Table 2 and plotted in Figure 2, along with data from other aluminium-FM73 single lap joints of different geometries tested in constant amplitude fatigue and reported previously [14]. Fatigue testing was limited due to manufacturing constraints. However, it can be seen that the data are consistent with other joints of the same materials when the maximum fatigue load is normalised against the joint static failure load. This provides additional reassurance of the validity of this data points. 
The expected variation of backface strain throughout the test depends on how the damage evolves at both ends of the joint. If the damage is balanced then as the damage front approaches the strain gauge the backface strain will rise to a peak value, when the damage is adjacent to the gauge and then decrease gradually as the damage progresses past the gauge until the joint finally fails. The rise in backface strain clearly depends on where the gauge is placed as this effects the initial value of the strain. Referring to Figure 3 it can be seen that the initial strain is lower the further the gauge is away from the overlap end. Thus the largest rises in strain will occur in the gauges furthest from the overlap ends.

Figure 3 and Figure 4 show the SG readings from Specimen 1, which was tested at 50\% of $\tau_{\mathrm{S}}$ and had a fatigue life of around 10000 cycles. The pattern at both ends is similar, showing 2 phases. During the first phase the change in strain is gradual. This can be considered as the nucleation or initiation phase of damage. Then, at around 7000 cycles (70\% of life), the backface strain rises more sharply until failure. As discussed above the change in strain in the gauges placed at 3 and $5 \mathrm{~mm}$ is more obvious than in the gauge placed at $1 \mathrm{~mm}$.

The rate of damage propagation through the joint can be seen from such data.

\section{Considering}

Figure 3 it can be seen that the gauge at $1 \mathrm{~mm}$ reaches a clear peak (and then decreases as the damage continues). Then, a little later in the test the damage reaches the gauges at 
$3 \mathrm{~mm}$, however it would appear that sudden failure occurred as damage reached the gauge at $5 \mathrm{~mm}$ as there is some evidence of a peak appearing just before final failure. Similar patterns can be seen in Figure 4, although there are no clear peaks in the gauges at 3 and $5 \mathrm{~mm}$ into the overlap. This could be caused by some asymmetry in the damage, which propagated more quickly at the end with gauges 1-3 than at the other end. All of the joints tested showed this trend, with damage being slightly greater at one end than the other.

The initial strain values for corresponding gauges are very similar at both ends of the joints. The small differences that occur can be attributed to inaccuracies when installing the SGs and small eccentricities induced by clamping the SLJ in the grips.

Strain data from one end of a second joint tested with the maximum cyclic load at $50 \%$ of $\tau_{s}$ is shown in Figure 5. The trend is similar to that seen in Specimen 1, and the fatigue life is very similar. The initial backface strain values measured by the SGs match well. Compared with the previous case even more damage occurred at end A with even the strain gauge at $5 \mathrm{~mm}$ exhibiting a peak. At the other side the trend was very similar to that seen in Specimen 1, where the damage is triggered by the damage initiating and expanding in the other end.

Figure 6 shows strain data at one end of a specimen (Specimen 3) that was tested with the maximum cyclic load being $40 \%$ of $\tau_{s}$ and had a fatigue life of around 60000 cycles. This gives evidence of more rapid fatigue damage growth at an earlier stage in the fatigue life. Interestingly, by considering the load-life data in Figure 2 it can be seen that the life of 
this specimen is reasonable consistent with the other joint data. The gauge at $1 \mathrm{~mm}$ reaches a peak fairly early on in the life. The gauge at $3 \mathrm{~mm}$ reaches a peak at about midlife whilst the gauge at $5 \mathrm{~mm}$ seems to be reaching a peak (i.e. damage adjacent to it) when the joint fails. The strain readings at the other end are similar to those seen in Figure 4, with none of the strains reaching a peak. This implies that damage was more widespread at the end of the joint containing the strain data shown in Fig 6.

Specimen 4 was tested with a maximum cyclic load of $30 \% \tau_{\mathrm{S}}$ and had a fatigue life of around 430000 cycles. The strain data from the more severely damage end is shown in Figure 7. The damage seems to have occurred less rapidly than in the specimen tested at $40 \%$ (Fig 6) and is more consistent with the data shown for the 50\% tests (Figs 3 and 5). The gauges reached a peak between 150000 and 250000 cycles in an appropriate order (ie $1 \mathrm{~mm}$ then $3 \mathrm{~mm}$ and finally $5 \mathrm{~mm}$ into the joint). The fact that the strains in the gauge $5 \mathrm{~mm}$ into the joint reached a peak and then reduced over a considerable portion of the life suggests that there was considerable damage propagation passed $5 \mathrm{~mm}$ into the joint and that sudden failure did not occur until the damage was more advanced. As final failure is a static failure this would be expected as the maximum applied load is considerably lower and thus a smaller undamaged adhesive zone is needed to sustain these reduced loads. Unlike the joints tested at 50\% where transitions regions were evident in all gauges, transition regions can only be seen in the gauges at 3 and $5 \mathrm{~mm}$ into the joint. 
As with the other joints the damage at the other end was less extensive, with no gauges reaching a peak. They exhibited a similar trend to that shown for the joint tested at $50 \%$ and illustrated in Figure 4.

The data obtained from these specimens shows some of the advantages of the backface strain technique. Using this technique it was possible to determine where the damage initiated and how it propagated along the adhesive. This represents a big advantage when compared to other monitoring methods and provides extensive data that can be used in the development of fatigue damage models.

\section{The damage model}

From earlier studies involving sectioning of partially fatigued joints [14] it was apparent that a zone of damaged developed in adhesive with increasing fatigue cycles. One way of representing this damage is to degrade the constitutive properties of the adhesive, represented in this work as an elasto-plastic response. A cyclic damage model has been developed and this has been based on maximum fatigue strain and it is reasonable to assume that this will drive the fatigue damage. As far as the authors are aware this is the first time progressive adhesive fatigue damage has been modelled in this way. The elastoplastic damage model was developed in Fortran and is applied in ABAQUS as a user defined field. A flow chart of the model is presented in Figure 8. The damage rate is calculated at each adhesive material integration point using Equation 1, where $\triangle D / \Delta N$ is the cyclic damage rate and $\varepsilon_{\max }$ and $\varepsilon_{\text {th }}$ are the adhesive maximum principal strain and 
threshold strain respectively at that material integration point; $b$ and $z$ are parameters input when calibrating the damage model. In every increment the damage at every adhesive material integration point is updated using Equation 2, up to a limiting value of $\mathrm{D}=1$ (fully damaged).

$$
\begin{array}{r}
\frac{\Delta D}{\Delta N}=b \times\left(\varepsilon_{\max }-\varepsilon_{t h}\right)^{z} \\
D_{i}=D_{i-1}+\left[\left(\frac{\Delta D}{\Delta N}\right) \times \Delta N\right]
\end{array}
$$

As the damage progressed the elastic modulus reduced from 2000 (undamaged) to $2 \mathrm{MPa}$ (fully damaged). Similarly, the yield stress reduced from 64 to $6 \mathrm{MPa}$. Ideally these properties would be reduced to zero when fully damage but this produced numerical instabilities in the modelling and the parametes adopted here were large enough to avoid these problems without significantly affecting the predicted fatigue life. The number of cycles was proportional to the time step used in ABAQUS. Although the threshold strain has been included in the formulation a zero value has been used in this work (ie no threshold has been applied). Damage parameters $b$ and $z$ were adjusted to fit the experimental fatigue data. These data included the fatigue life and the backface strain response.

\section{The ABAQUS model}


At this stage modelling has mainly been undertaken in 2D as the solutions are non-linear and require considerable processing time. The SLJs were modelled in ABAQUS using 8 noded plane strain elements. The material model for the adhesive was describe above. The aluminium was modelled as a linear elastic material with Young's modulus and Poisson's ratio of 70GPa and 0.33 respectively. The yield stress of the aluminium was in excess of $300 \mathrm{MPa}$ and thus plasticity was not expected in the fatigue tests, where the maximum load was $50 \%$ of the static failure load. Following a mesh sensitivity study the mesh shown in Figure 9 was used. The boundary conditions modelled the joint as fully built in at one end with a force (maximum fatigue load) applied in the $\mathrm{X}$ (axial) direction at the other end together with a zero rotation and Y (vertical) displacement. These zero rotations at both ends of the joint represented the clamping in the grips of the test machine. The joint was only modelled between the grip ends.

\section{Parametric study}

Prior to calibrating the damage model with the experimental data, it was necessary to run a parametric study to assess the effect of the input parameters on the predicted fatigue response of the modelled joints. As a consequence multiple analyses were carried out with different $b$ and $z$ values. The effect of $b$ and $z$ was assessed not only by considering the backface strain, but also by considering other output variables like the damage and fatigue life. 
Preliminary analyses showed that parameter values of $b=0.00014$ and $z=0.5$ gave an appropriate value of fatigue life at the case where the applied load is $40 \%$ of $\tau_{s}$ thus ensuring that parameter values were representative This parameter set formed the starting point for the parametric study,. The evolution of the strain distribution along the substrate backface in the overlap region for these parameters is shown in Figure 10 in the form of strain distributions after various fatigue cycles. As the model is symmetric the induced damage is symmetric and hence equal at both ends. Thus it is only necessary to consider strains on one substrate. It can be seen from Figure 10 that the backface strain peak, that was initially at around $30 \mathrm{~mm}$ (i.e. the overlap end) shifted along the overlap as the damage (and number of cycles) increased. By considering the strain at a certain position along the overlap (say $25 \mathrm{~mm}$, corresponding to the gauge $5 \mathrm{~mm}$ inside the overlap) it can be seen that the strain starts as positive, decreases, becomes negative and finally reach a peak value that is largely unchanged with further fatigue damage. These are the trends in the backface strain data reported and discussed in the experimental section of this paper.

This type of graph was also very helpful when determining where to install the SGs. The most sensitive region in terms of slope is between 23 and $29 \mathrm{~mm}$. This means that as the damage increases, there will be a larger change in strain than at other locations, thus it will be easier to detect and monitor.

The backface strain patterns at the nominal SG locations are shown in Figure 11. These have been found by considering the change in backface strain at a fixed location on the 
substrate (1, 3 and $5 \mathrm{~mm}$ into the overlap) from the data shown in Fig 10. The trend is very similar to that measured during the experimental tests (e.g. Fig 3). The SG placed $1 \mathrm{~mm}$ inside the overlap records a moderate change in strain, and after reaching a peak at 40000 cycles, decreases until failure, at 58000 cycles. The pattern in the gauges at 3 and $5 \mathrm{~mm}$ is very similar, in both there is a clear transition at 33000 cycles. The main difference lies that the joint fails after reaching the peak strain at $5 \mathrm{~mm}$, while the one at $3 \mathrm{~mm}$ does measure a decrease during the final 12000 cycles. As mentioned in the experimental section, this final sudden failure is a result of the remaining undamaged adhesive being unable to sustain the maximum fatigue load resulting in collapse of the joint. Differences in initial strain between the experimental and modelled data (shown in Fig 10 and later figures) can, in part, be attributed to errors in the positioning of the gauges and strains induced whilst clamping the specimen in the grips. Although care was taken these effects cannot be minimised.

Damage evolution for this same analysis is shown in Figure 12. The damage is distributed across the overlap but was higher at the adhesive edges, where it propagated at a faster pace. After 3920 cycles the damage in the edges is 0.21 , while in the centre region is only 0.069 . At 28420 cycles these values are 0.932 and 0.25 . This represents an increase of $343 \%$ and $204 \%$ respectively. At the end of the test all of the adhesive exhibits significant damaged ( $>0.5$ ) whilst at the overlap ends it has failed (full damage, $\mathrm{D}=1$ ) for a length of $5 \mathrm{~mm}$ at each end of the overlap. Although the fully damaged zones seem fairly consistent with the backface strain plots it is unclear whether the actual 
experimental damage in the remaining adhesive is as widespread as that predicted (Figure 12).

The effect of increasing $b$ is very simple, it just accelerates the damage rate. Thus when $b$ was increased from 0.00014 to 0.00030 the fatigue life decreased to 28000 cycles with no major changes seen in the backface strain or damage evolution, the initial and final values in both cases are similar

The effect of $z$ is more complex. The maximum principal strain throughout the adhesive is less than 1, thus if it is raised to a higher power it will give a lower damage rate, increasing the fatigue life. The damage will also localise more at the overlap ends as raising to a higher power accentuates the difference between the low strain in the interior of the overlap the higher strains at the overlap ends. Figure shows the damage evolution for an analysis run at $z=8$. The $b$ parameter had to be increased to $2 \times 10^{8}$ to give a representative fatigue life. This illustrates the large influence of $z$ on the damage model. The damage evolution is very different from the analysis with $z=0.5$. The damage was more focused at the overlap ends and in this region the adhesive became fully damaged after only 4000 cycles. The damage at the centre is much lower, and even at the end of the simulation there remained a region of undamaged adhesive. On the other hand the zone of fully damaged adhesive is approximately $24 \mathrm{~mm}$, considerably higher than with the lower $\mathrm{z}$ value. 
The backface strain evolution at this higher $z$ value (Figure ) was notably different to that at the lower $\mathrm{z}$ value (Figure 11).With the increased $\mathrm{z}$ value the strain peaks occur much earlier in, and are more spread out over, the fatigue life of the joint. This is attributable to the more localised nature of the damage that occurred in this analysis.

So far only the data at one level of fatigue loading has been considered. By choosing a parameter set that matches the fatigue life over a range of fatigue load levels a unique set of parameters, for this fatigue damage model, can be determined. This can be considered as a calibration process and is discussed in the next section..

\section{Calibration}

This process was achieved by finding the parameter $b$ that provided the correct fatigue life at $40 \%$ for a range of $\mathrm{z}$ values. These parameter sets were then used to prodict the fatigue lives at 50\% and 30\% fatigue loading. The predicted load-life data for 3 values of $\mathrm{z}$ together with the experimental data are shown in Fig 15.. It can be seen that a high value of $z=8$ was necessary to fit the fatigue lives at $50 \%, 40 \%$ and $30 \%$ of $\tau_{\mathrm{S}}$. This calibration was performed by trial and error and the optimum value of $b$ ( with $\mathrm{z}=8$ ) was found to be $3.5 \times 10^{8}$. The backface strain variations and lives were compared to the corresponding data measured in the tests as discussed below.

Figure 16 shows a comparison between the measured and predicted backface strain for Specimen 1, tested at $50 \%$ of $\tau_{\mathrm{S}}$ (Fig 3 ). The fatigue lives matched very well, both being approximately 10000 cycles. On the other hand the transition in the backface strain data 
occurred a little earlier in the simulations than in the actual experiment. In any case the initial and final backface strain values match well.

The match for Specimen 4, tested at $30 \%$ of $\tau_{\mathrm{S}}$ (Fig 7), where the damage initiated and expanded more quickly, was excellent (Fig 17). The life predicted by the damage model and backface strain pattern fit very well, especially the gauges at 3 and $5 \mathrm{~mm}$ into the overlap. The initial and final backface strain values in these 2 gauges are very similar for the experimental and damage model. There is an increase from the start of the test followed by stabilisation when the peak is reached.

The fit for specimens tested at $40 \%$ of $\tau_{\mathrm{S}}$ reasonable but not as good as the other specimens. Figure 18 shows the match for SG1 and 2. The trends are fairly similar but the fitting is effected by the difference in life, the experimental tests lasted 60000 cycles while the damage model only predicted a life of 40000 cycles. However, fatigue data often experiences considerable scatter and the predicted data could easily lie within this range. A summary of all tests carried out at the University of Surrey is shown in Figure 2, and it can be seen that the damage model life predictions fit the global trends very well.

\section{Conclusions}

The SLJs tested at different load levels in constant amplitude fatigue gave reliable and consistent load-life data. The backface strain profiles obtained from the gauges depended on the load applied. At higher loads the life was shorter, and the backface strain trend was 
an initial region of moderate change, followed by an acceleration (transition) until reaching a peak. The joint tended to fail as the gauge at $5 \mathrm{~mm}$ approached its peak value.

At lower fatigue loads the joints can sustain more extensive damage before final failure as the applied level of loading is lower and thus less overlap is required to sustain this load. This can be demonstrated by the more extensive backface strain profiles developed at lowere loads with the transition region occurring at a relatively earlier part of the fatigue life.

An elasto-plastic damage model has been developed, capable of matching backface strain and fatigue life patterns. This represents a significant achievement. The damage model relies on decreasing the elastic modulus and the yield stress as the damage propagates. Output data were compared to experimental data taken at $50 \%, 40 \%$ and $30 \%$ of $\tau_{\mathrm{S}}$. The backface strain match was good and the fatigue life predictions were within scatter. The parametric study showed that a high $z$ was necessary to fit the fatigue life over the range of load levels considered.

The use of six strain gauges in staggered configuration was a very powerful tool in monitoring fatigue initiation and propagation. This method is capable of locating fatigue initiation with accuracy. It was also capable of discriminating the damage profiles at both ends, showing where it initiated and which side was damaged as a consequence. Videomicroscopy may be a useful complement but should remain as a secondary technique, as it only monitors a small exterior region of the adhesive overlap. 


\section{References}

[1] Quaresimin, M., Ricotta, M. Comp. Sci. and Tech. 2006; 66: 176-187.

[2] Xu, XX., Crocombe, AD., Smith, PA. J. Adhesion 1996:58 (3-4): 191-204.

[3] Apalak, MK., Engin, A. J. of Adh Sci and Tech 2004: 18 (5): 529-559.

[4] Dessureault, M., Spelt, JK. Int. J. of Adh and Adhes 1996; 17 (3): 183-195.

[5] Ishii, K., Imanaka, M., Nakayama, H., Kodama, H. (1998), Comp. Sci. and Tech.1998; 59: 1675-1683.

[6] Zeng, Q., Sun, T.C. Fatigue. Fract. Eng. Mat. 2004; 27: 413-422.

[7] Cheuk, P.T., Tong, L., Wang, C.H., Baker, A., Chalkley, P . Comp. Struc 2002; 57: 109-115.

[8] Zhang, Zh., Shang, J.K., Lawrence, FV. J. Adhesion 1995; 49: 23-26.

[9] Crocombe, A.D., Ong, C.Y., Chan, C.M, Wahab, MMA. J. Adhesion 2002; 78 (9): 745-776.

[10] Lefebvre, D.R, Dillard, D.A J. Adhesion 1999; 70 (1-2): 119-138.

[11] Imanaka, M., Hamano, T., Morimoto, A., Ashino, R., and Kimoto, M. J. of Adh. Sci. and Tech. 2003; 17 (7): 981-994. 
[12] Crocombe, A.D., Richardson, G. Int. J. of Adh. and Adhes. 1999; 19: 19-27.

[13] Barrandon, T.(2004), Fatigue tests on single lap joints using the backface strain method, University of Surrey.

[14] Graner Solana, A., Crocombe, A.D, Wahab, M.M.A, Ashcroft, I.A, J. Adhesion Sci. and Technol.2007; 21- 14: 1343-1357. 


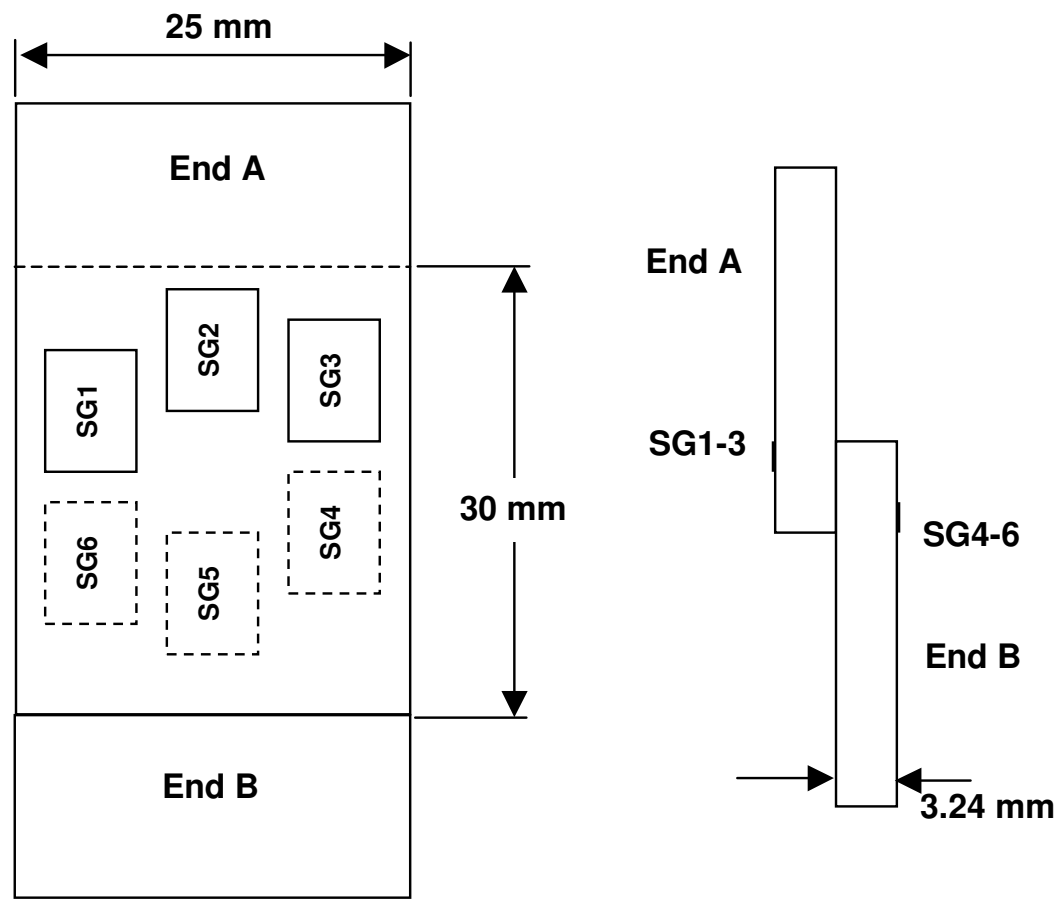

Figure 1: SG positions in adhesive overlap (not to scale).

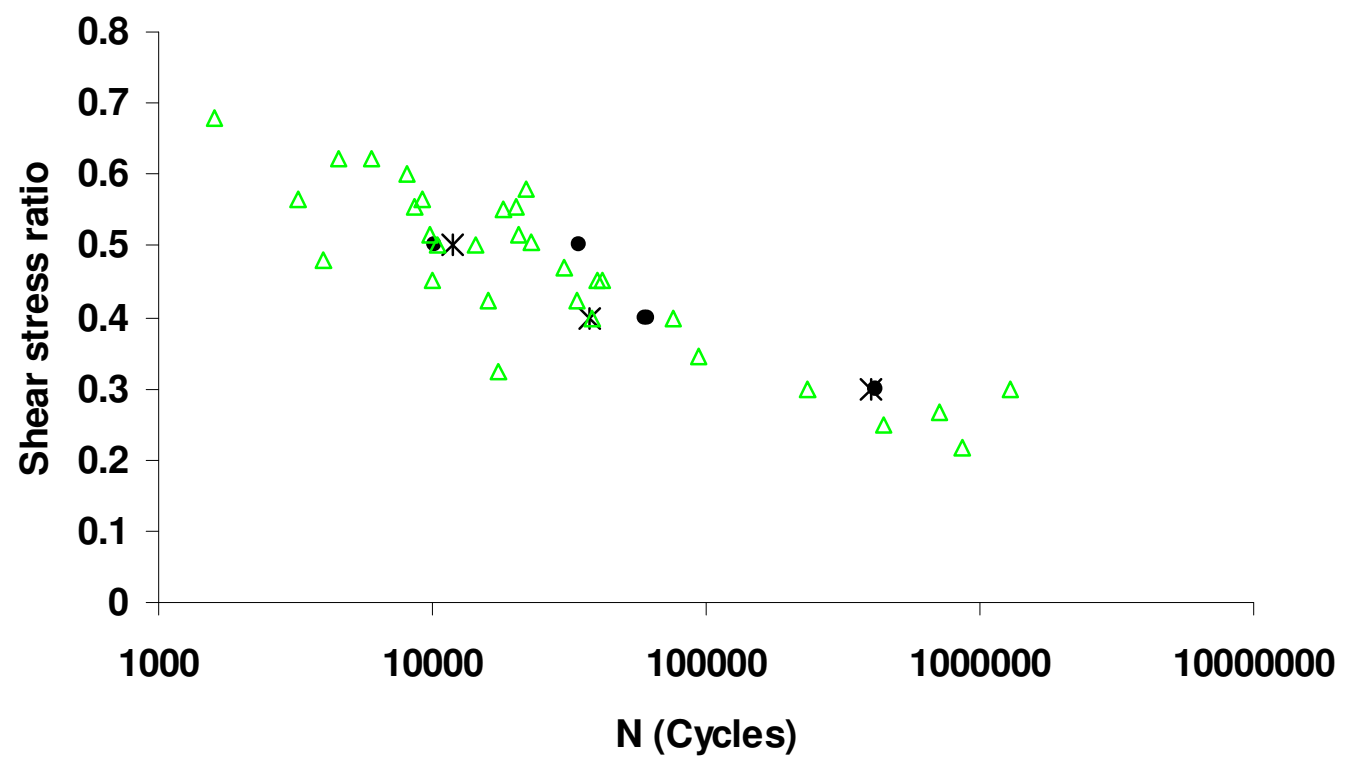

- Experimental tests $*$ Damage model $\triangle$ Other tests

Figure 2: Fatigue load-life experimental data and comparison with calibrated damage model predictions. 


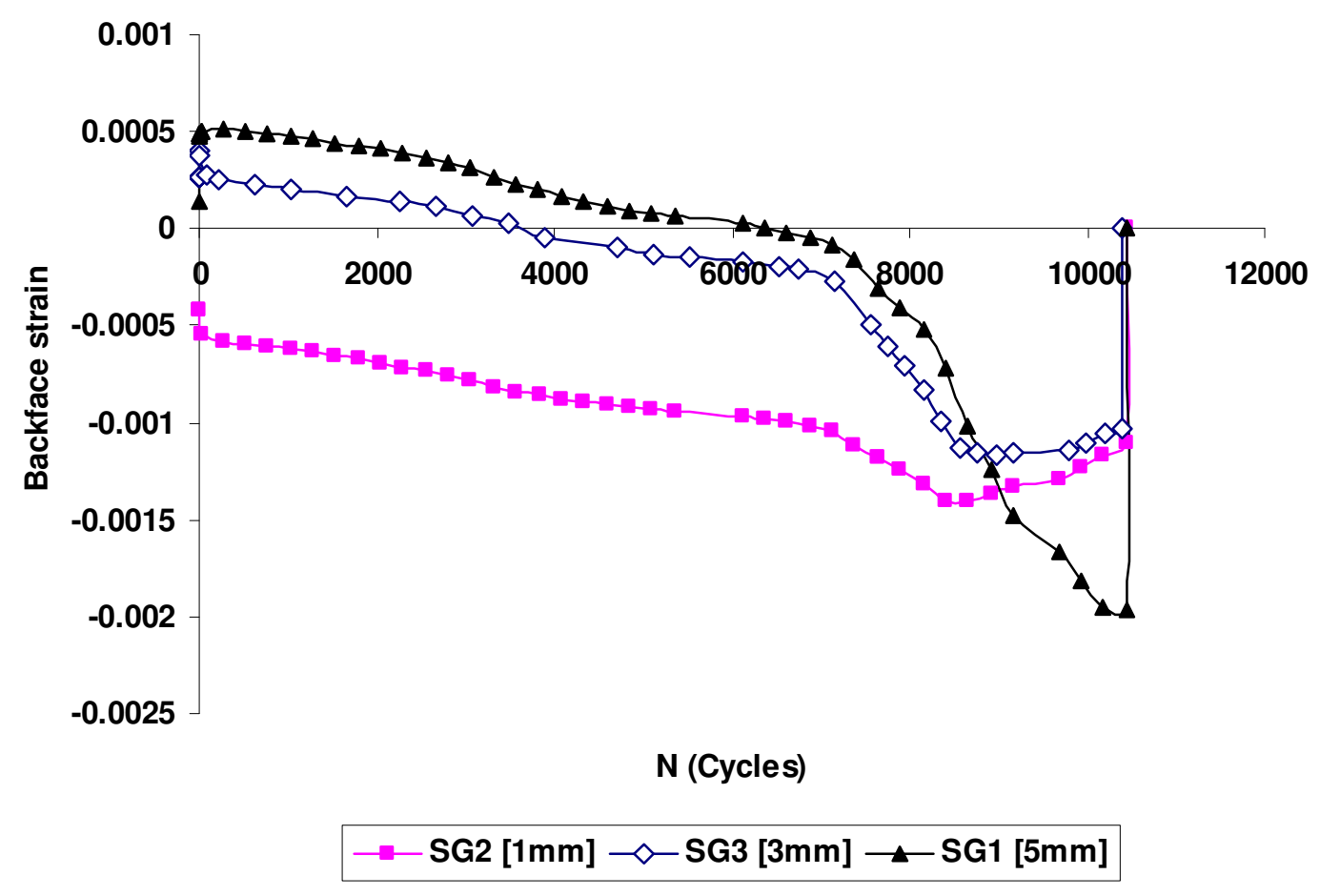

Figure 3: Specimen 1 tested at $50 \%$ of $\tau_{S}$ (SG1-3).

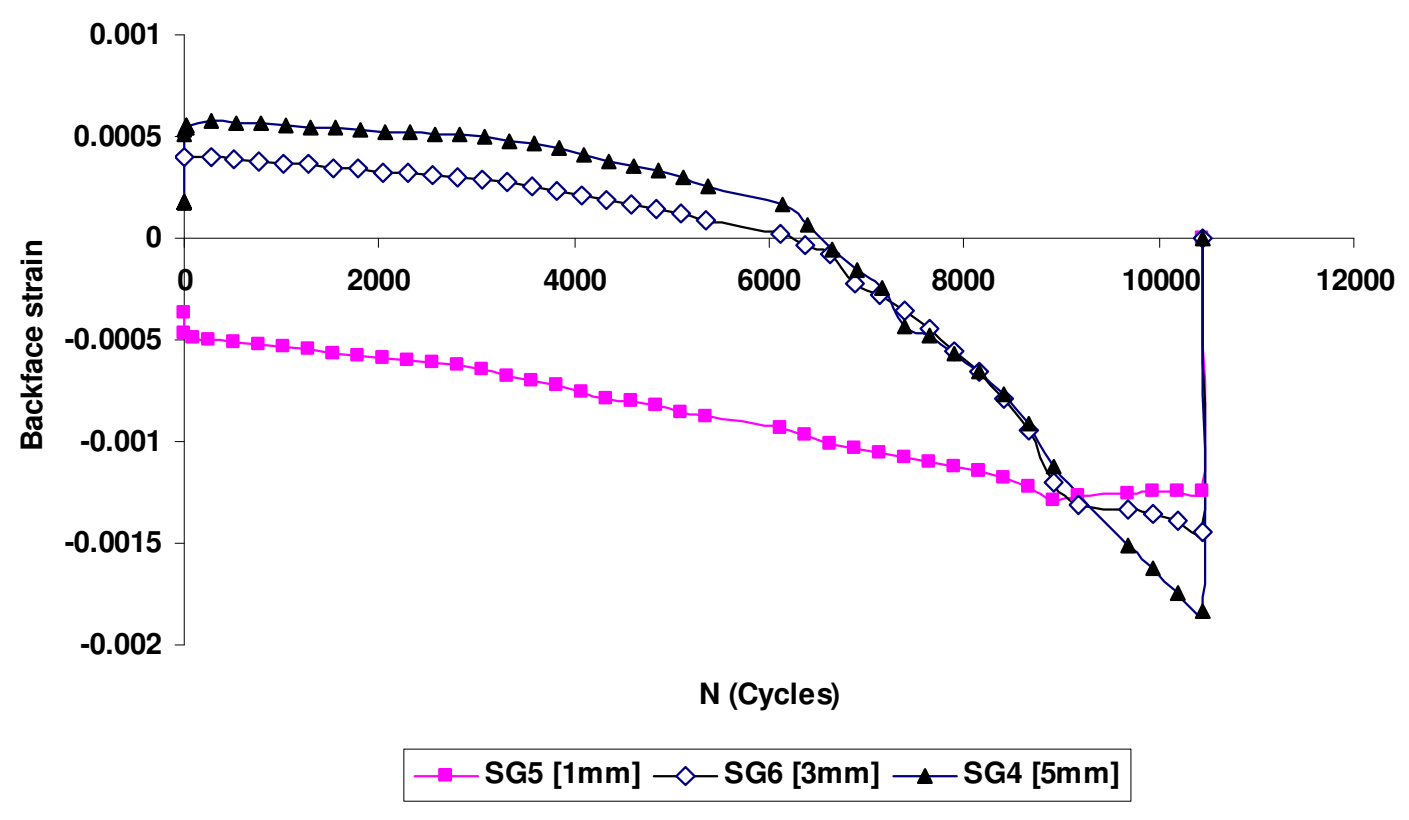

Figure 4: Specimen 1 tested at $50 \%$ of $\tau_{S}$ (SG4-6). 


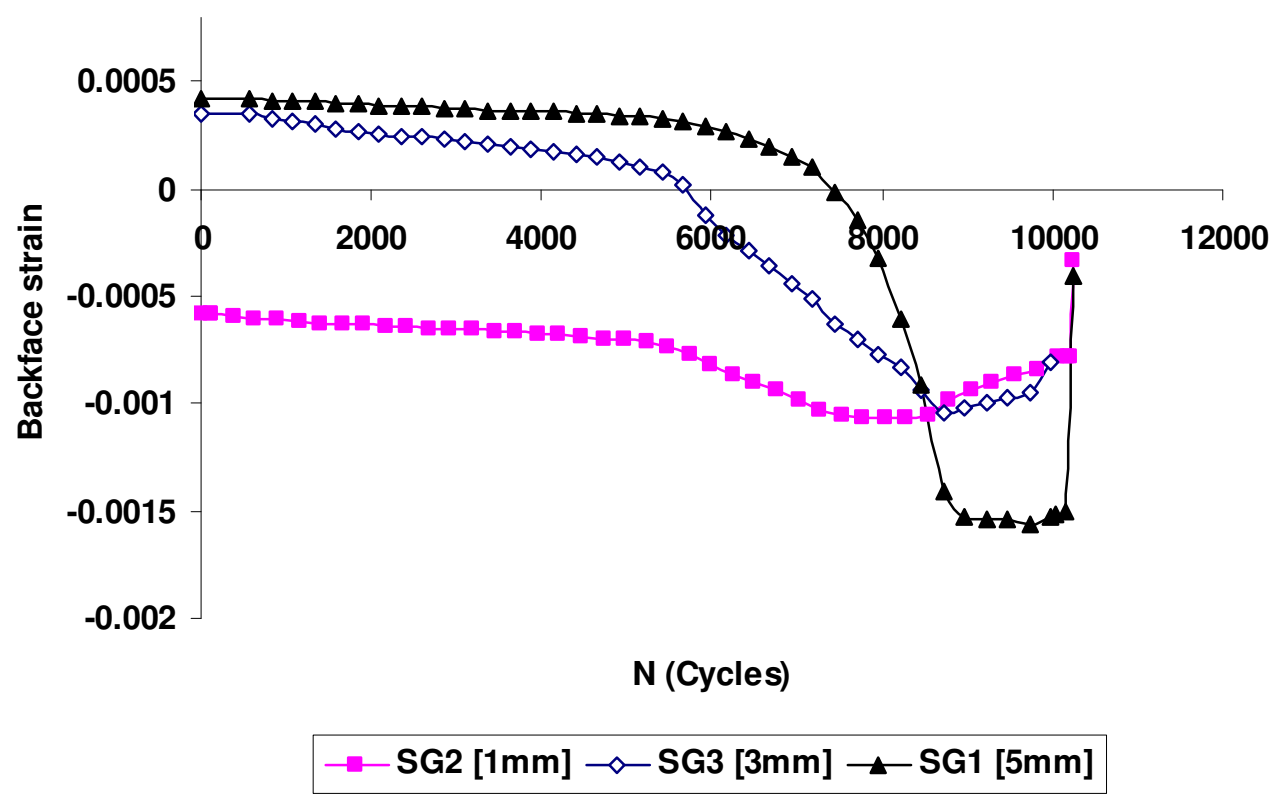

Figure 5: Specimen 2 tested at $50 \%$ of $\tau_{S}$ (SG1-3).

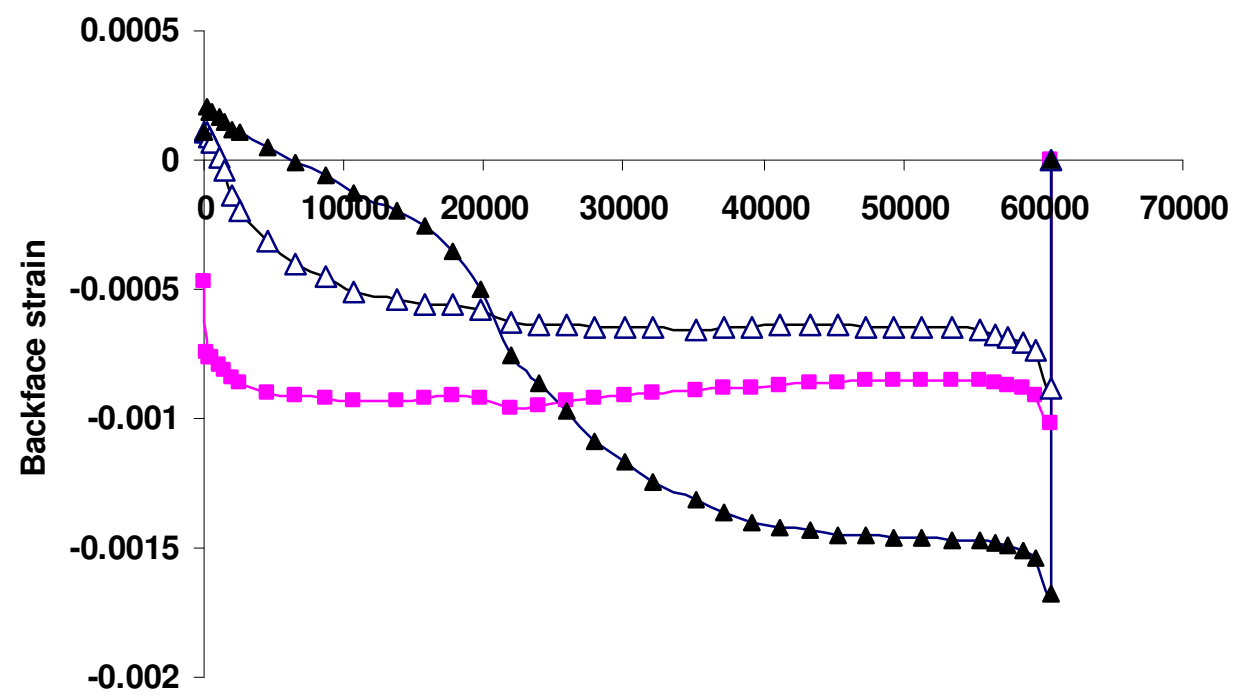

N (Cycles)

$\neg \mathrm{SG} 2[1 \mathrm{~mm}] \neg \mathrm{SG} 3[3 \mathrm{~mm}] \multimap \mathrm{SG} 1[5 \mathrm{~mm}]$

Figure 6: Specimen 3 tested at $40 \%$ of $\tau_{S}$ (SG1-3). 


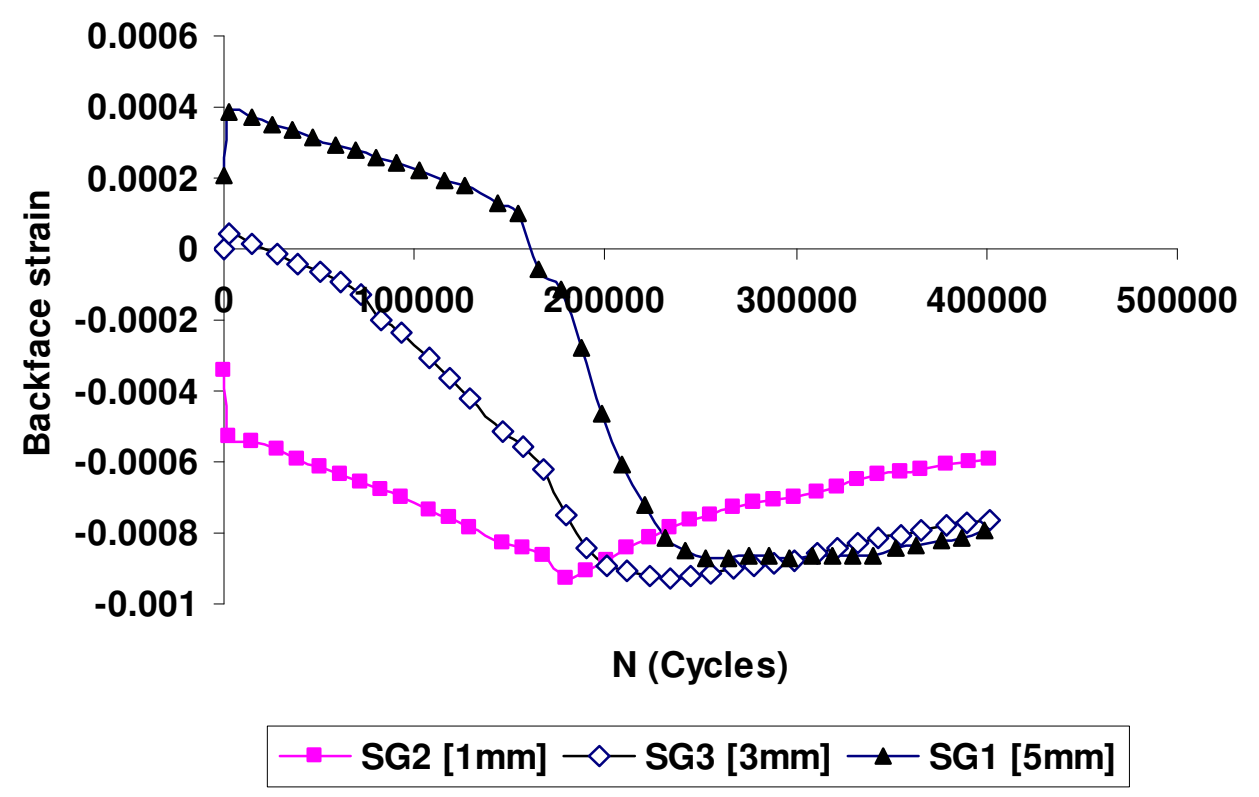

Figure 7: Specimen 4 tested at $30 \%$ of $\tau_{S}$ (SG1-3).

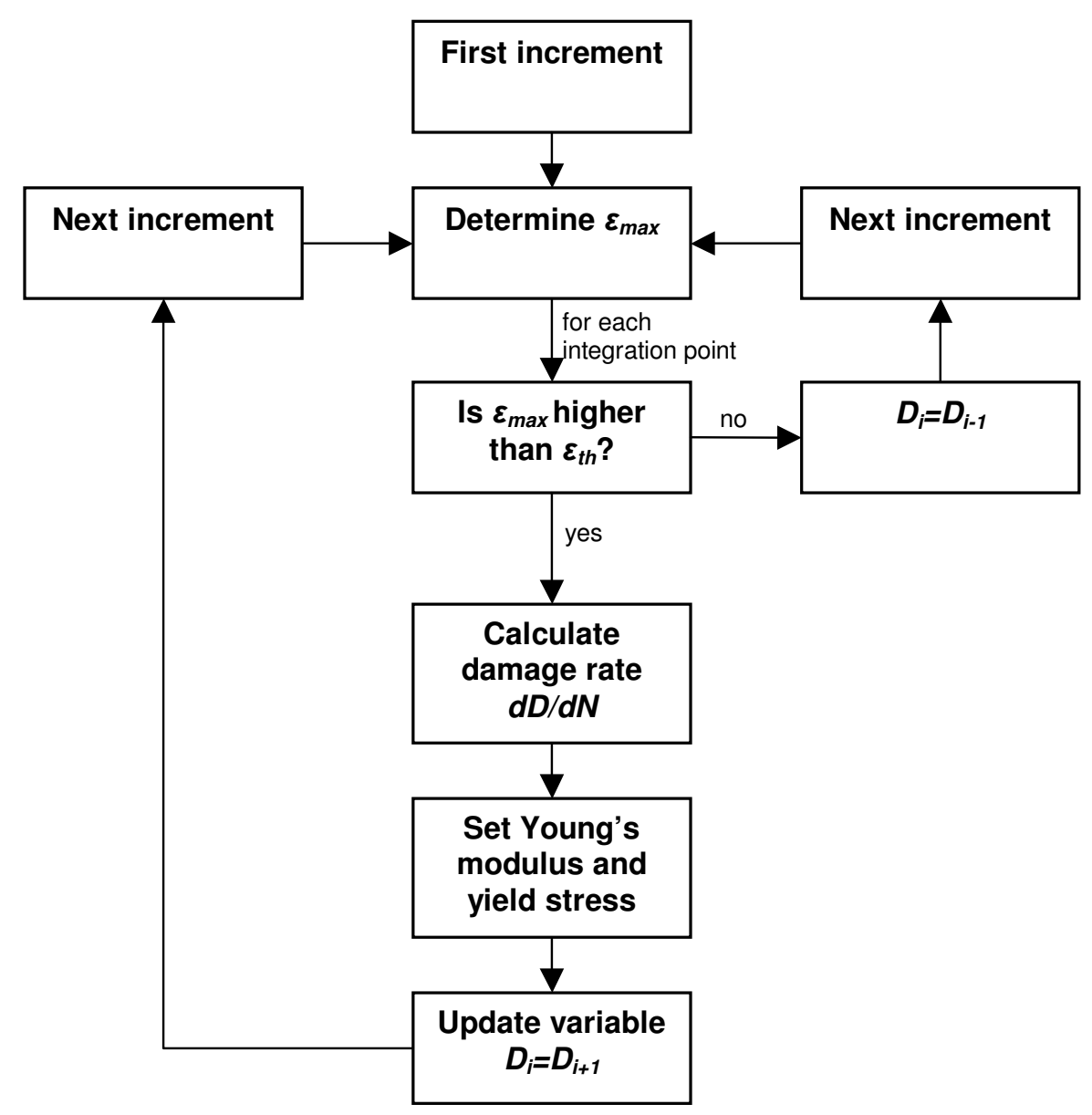

Figure 8: Flow chart of damage model. 


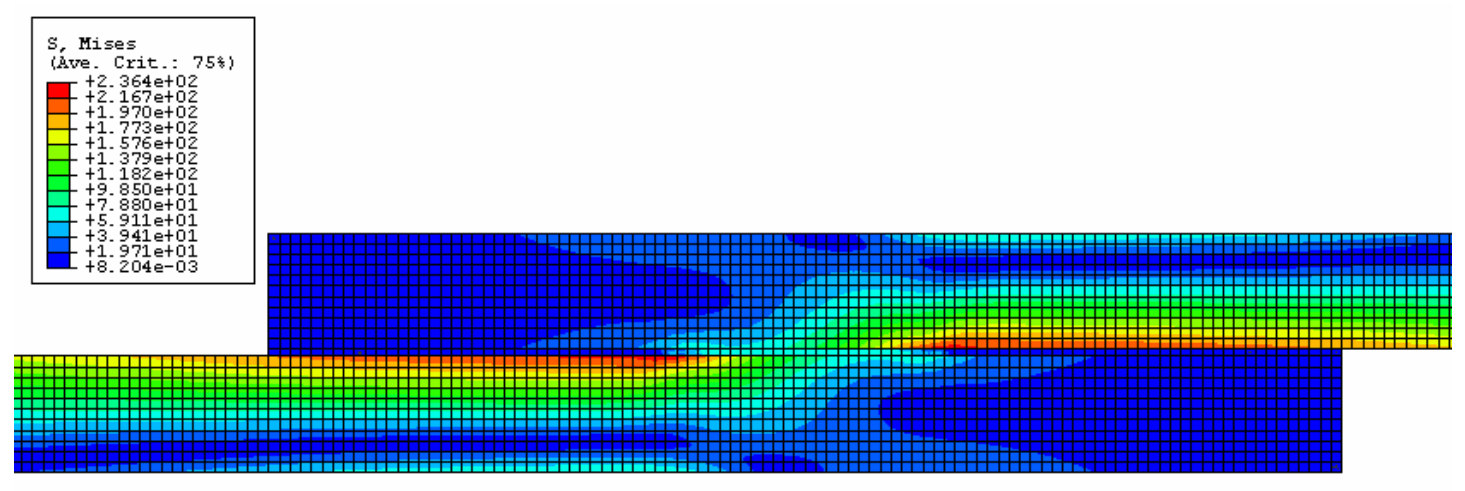

Figure 9: 2D mesh applied in fatigue simulations.

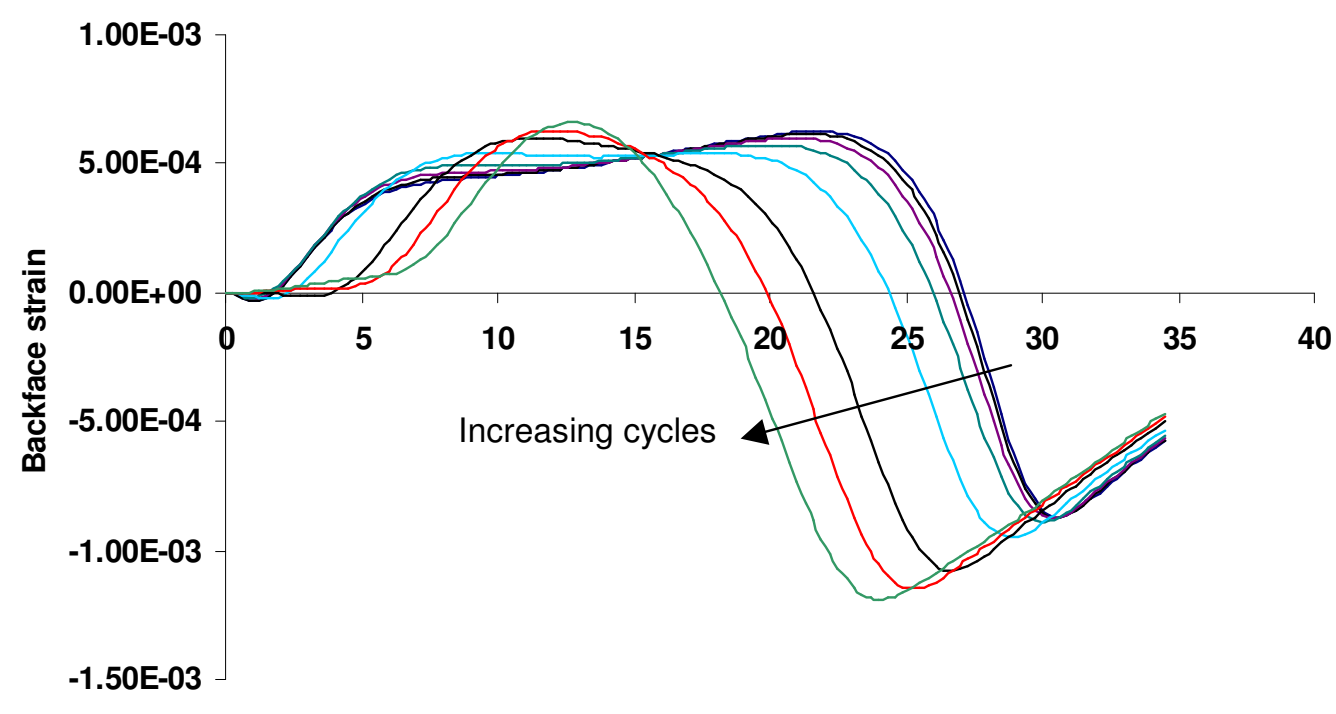

Distance from the substrate free end $(\mathrm{mm})$

$-0 \longleftarrow 8820-18620 \longleftarrow 28420-38220-48020-52920 \longleftarrow 57877$

Figure 10: Backface strain evolution on top of overlap at $40 \%$ of $\tau_{S}(z=0.5 b=0.00014)$. 


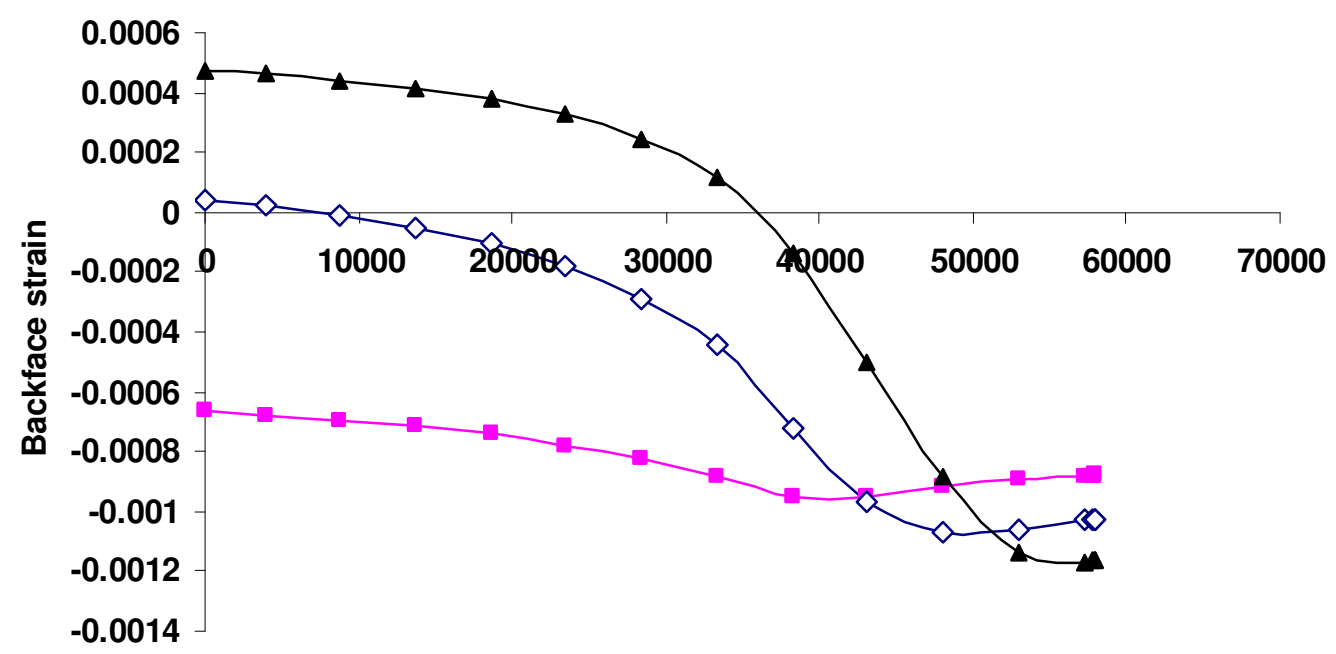

N (Cycles)

$\neg 1 \mathrm{~mm} \curvearrowleft 3 \mathrm{~mm} \multimap 5 \mathrm{~mm}$

Figure 11: Backface strain evolution in SG locations at $40 \%$ of $\tau_{S}(z=0.5 b=0.00014)$

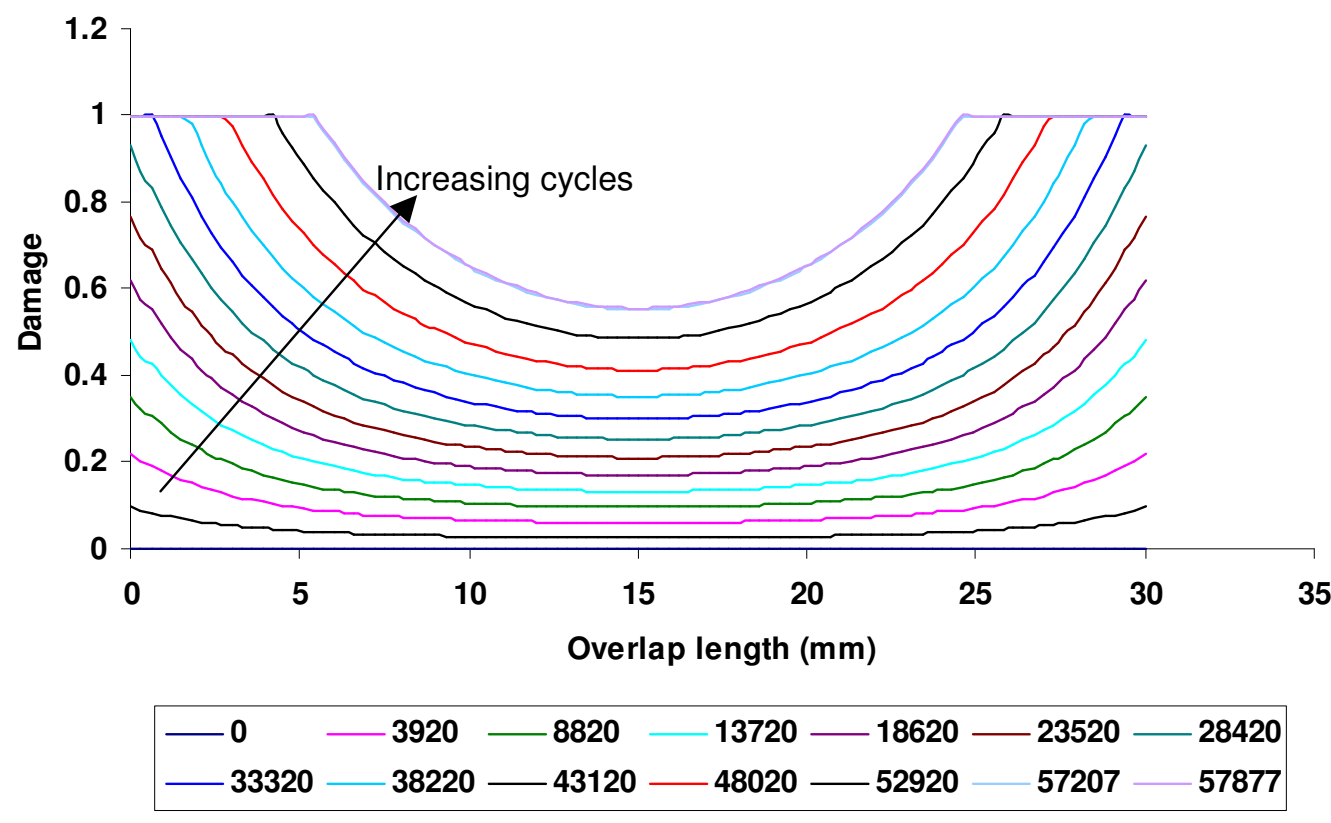

Figure 12: Damage evolution across adhesive at $40 \%$ of $\tau_{S}(z=0.5 b=0.00014)$ 


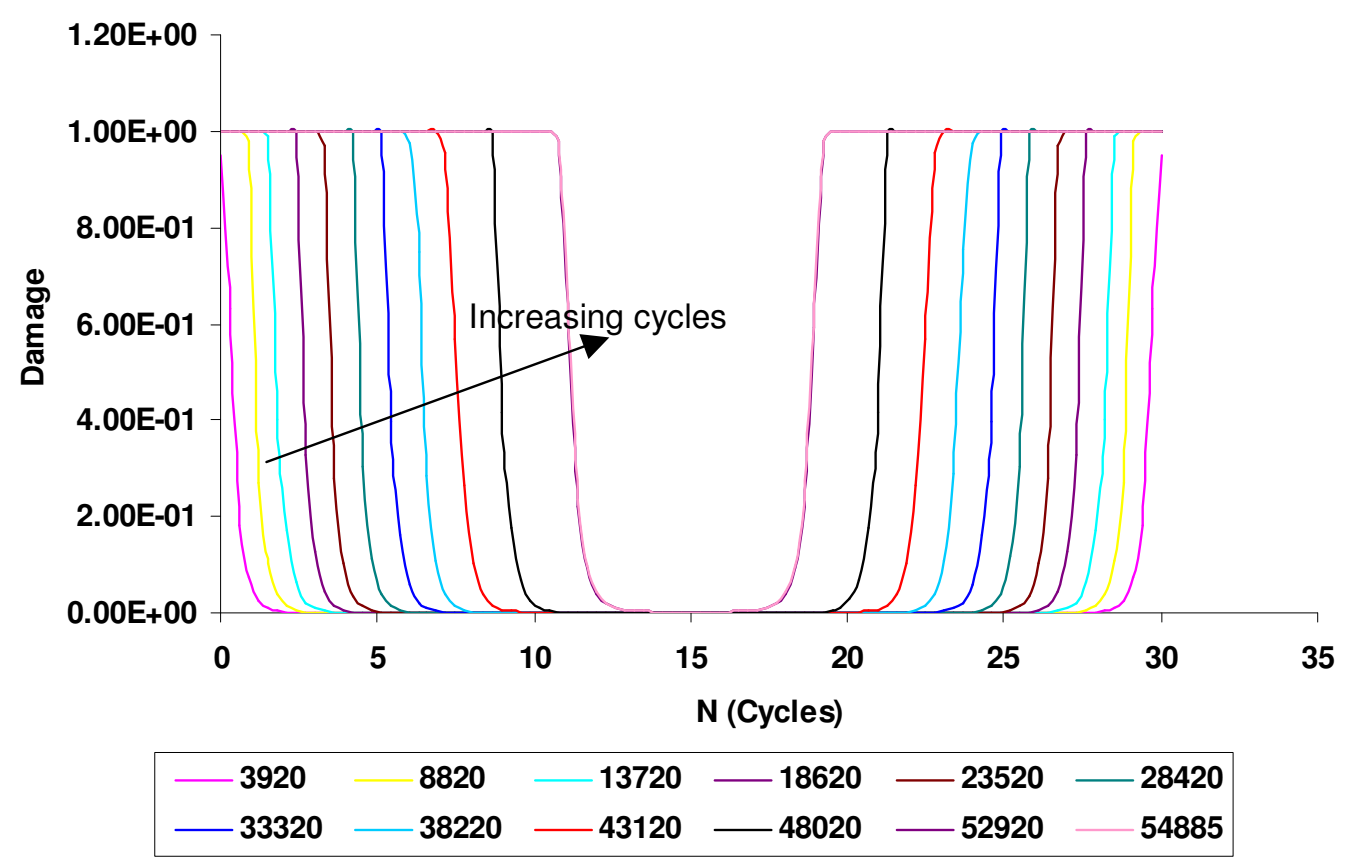

Figure 13: Damage evolution in SG locations at $40 \%$ of $\tau_{S}\left(z=8 b=2 \times 10^{8}\right)$ 


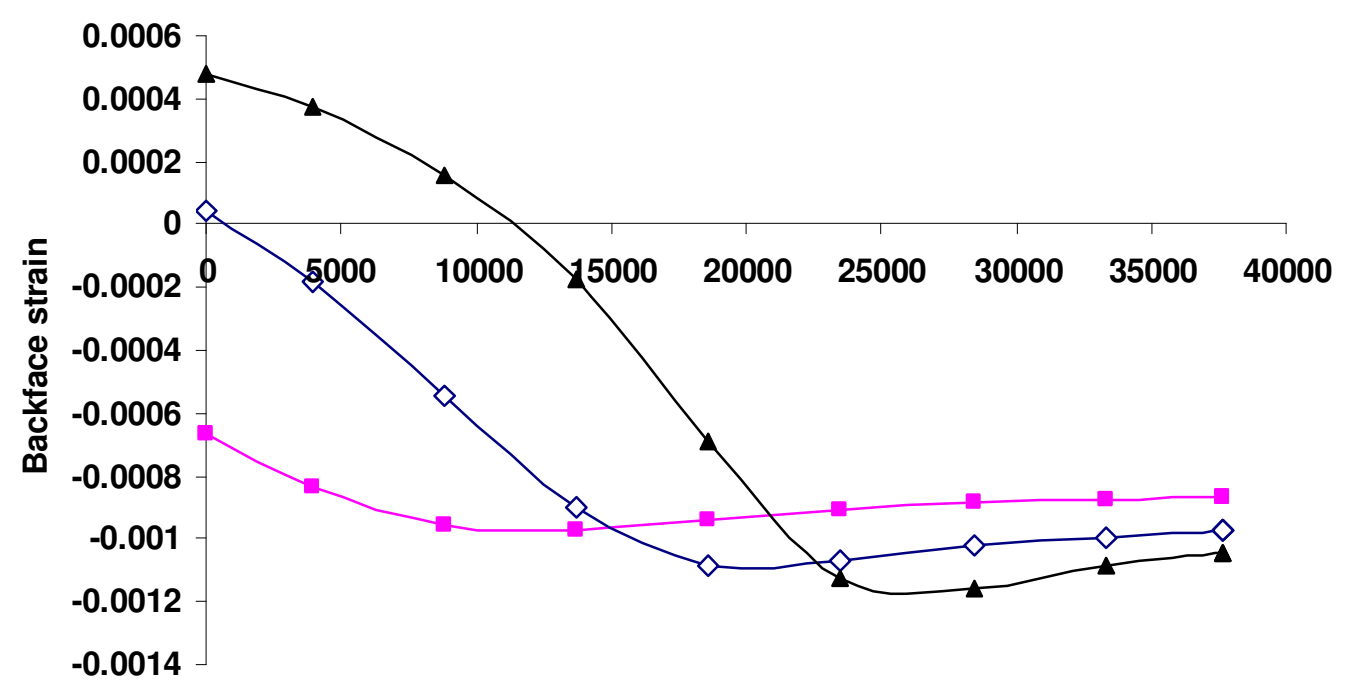

N (Cycles)

$\rightarrow-1 \mathrm{~mm} \leadsto-3 \mathrm{~mm} \rightarrow 5 \mathrm{~mm}$

Figure 14: Backface strain evolution in SG locations at $40 \%$ of $\tau_{S}\left(z=8 b=2 \times 10^{8}\right)$

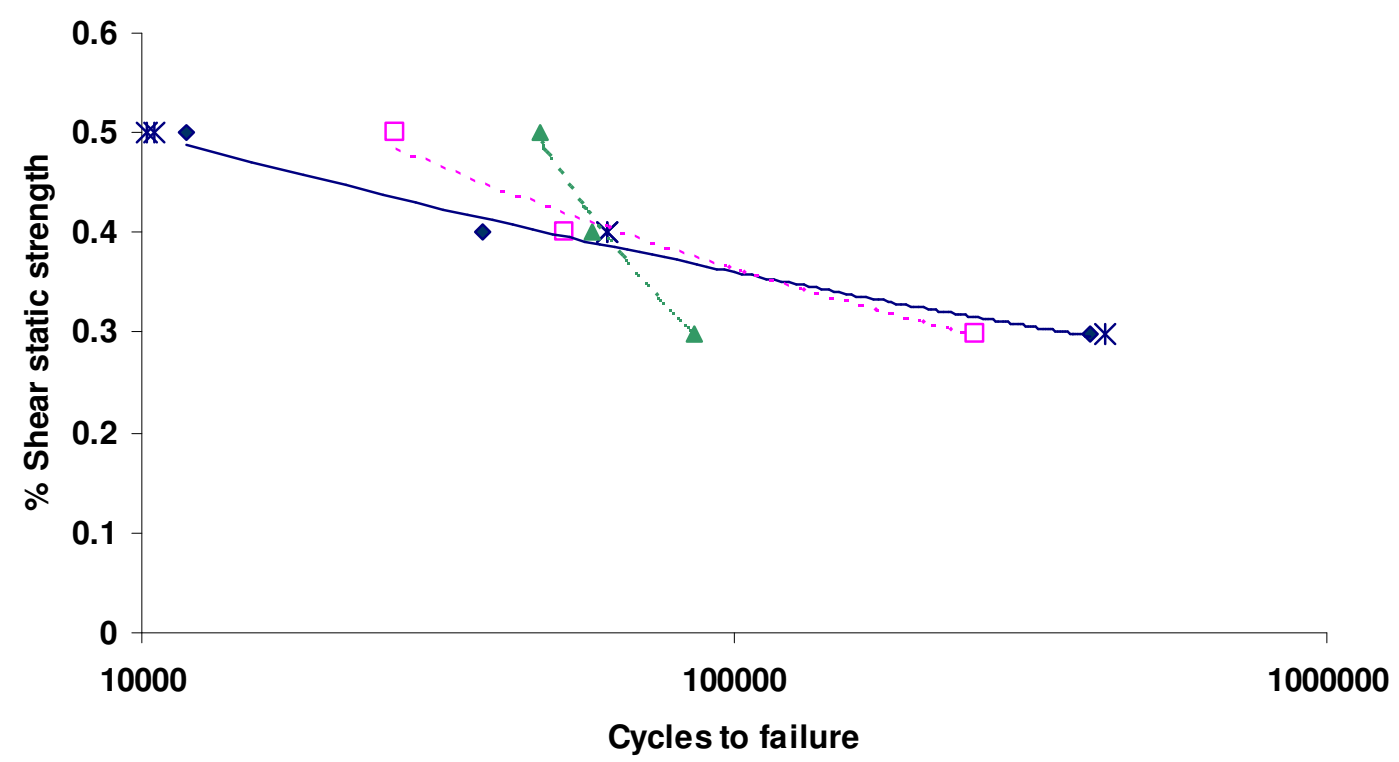

$*$ Experimental $\triangle \mathrm{z}=0.5 \quad \square \quad \mathrm{z}=4 \quad \bullet \quad \mathrm{z}=8$

Figure 15: Experimental compared to modelling Load-Life data. 


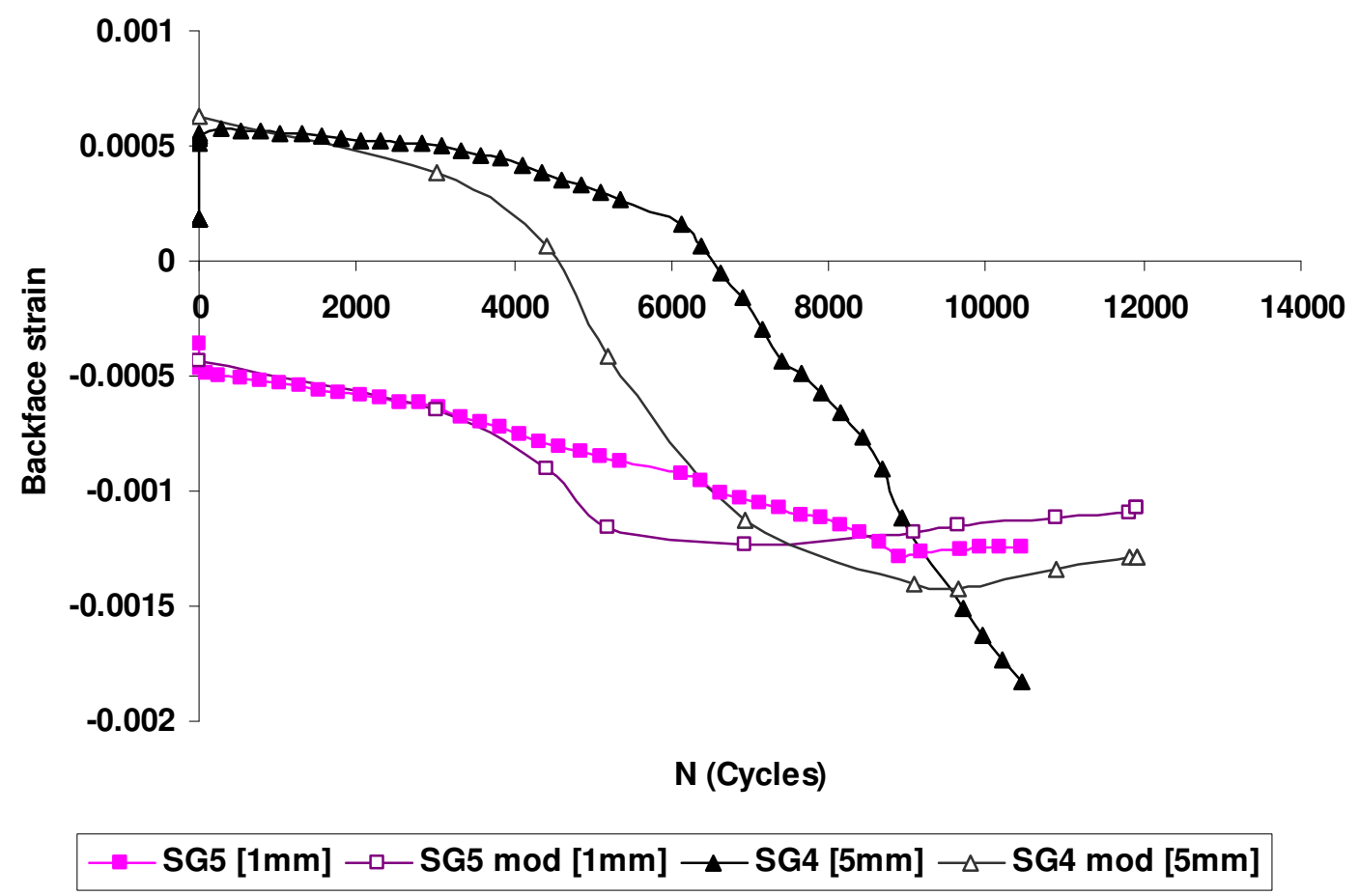

Figure 16: Experimental compared to modelling strain for different SGs, $50 \% \tau_{S}$

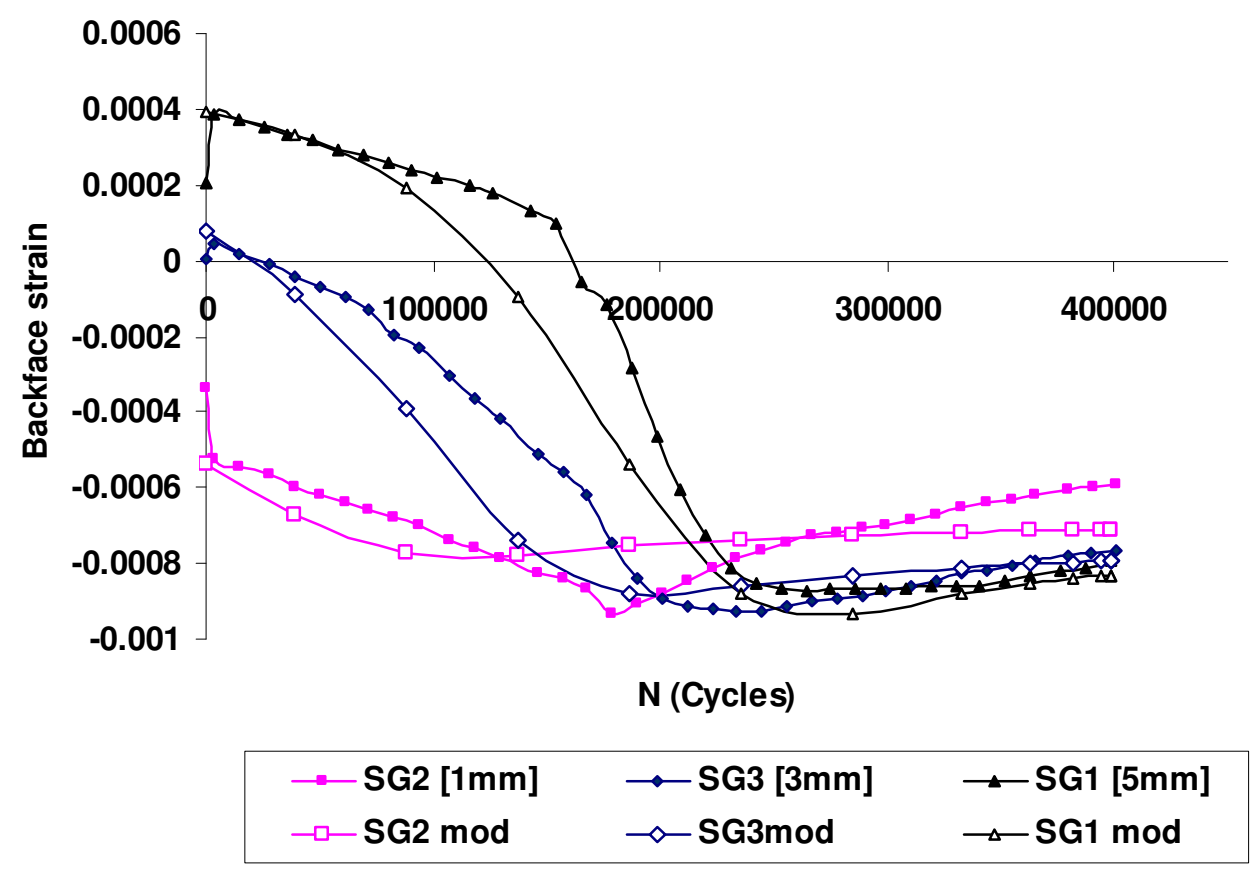

Figure 17: Experimental compared to modelling strain for different SGs, $30 \% \tau_{S}$ 


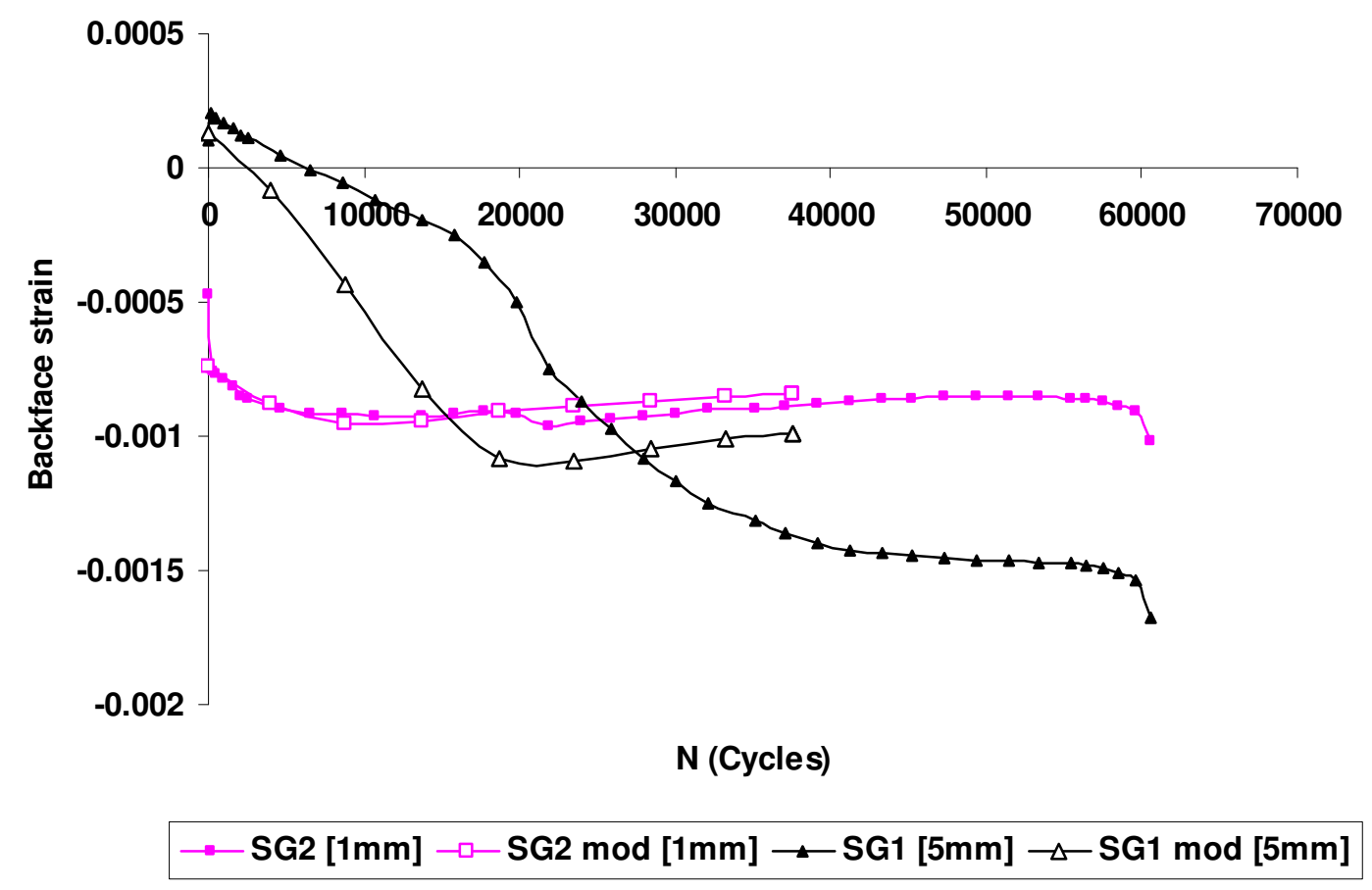

Figure 18: Experimental compared to modelling strain for different SGs, $40 \% \tau_{S}$ 
Table 1: Single lap joint characteristics.

\begin{tabular}{|c|c|}
\hline Type & Specification \\
\hline Aluminium & $2024-\mathrm{T6}$ \\
\hline Substrate length & $115 \mathrm{~mm}$ \\
\hline Substrate thickness & $3.24 \mathrm{~mm}$ \\
\hline Adhesive & FM-73M \\
\hline Overlap & $30 \mathrm{~mm}$ \\
\hline Adhesive thickness & $0.2 \mathrm{~mm}$ \\
\hline Width & $25 \mathrm{~mm}$ \\
\hline
\end{tabular}

Table 2: Experimental fatigue load-life data

\begin{tabular}{|c|l|}
\hline Maximum fatigue load/static failure strength & Life (cycles) \\
\hline 0.5 & $10442,10100,34253$ \\
\hline 0.4 & 60500,59995 \\
\hline 0.3 & 420000 \\
\hline
\end{tabular}




\section{List of Tables}

Table 1: Single lap joint characteristics.

Table 2: Experimental fatigue load-life data

\section{List of figures}

Figure 1: SG positions in adhesive overlap

Figure 2: Fatigue load-life experimental data and comparison with calibrated damage model predictions

Figure 3: Specimen 1 tested at $50 \%$ of $\tau_{S}$ (SG1-3)

Figure 4: Specimen 1 tested at $50 \%$ of $\tau_{S}$ (SG4-6)

Figure 5: Specimen 2 tested at $50 \%$ of $\tau_{S}$ (SG1-3)

Figure 6: Specimen 3 tested at $40 \%$ of $\tau_{S}$ (SG1-3)

Figure 7: Specimen 4 tested at $30 \%$ of $\tau_{S}$ (SG1-3)

Figure 8: Flow chart of damage model

Figure 9: 2D mesh applied in fatigue simulations

Figure 10: Backface strain evolution on top of overlap at $40 \%$ of $\tau_{S}(z=0.5 b=0.00014$

Figure 11: Backface strain evolution in SG locations at $40 \%$ of $\tau_{S}(z=0.5 b=0.00014)$

Figure 12: Damage evolution across adhesive at $40 \%$ of $\tau_{S}(z=0.5 b=0.00014)$

Figure 13: Damage evolution in SG locations at $40 \%$ of $\tau_{S}\left(z=8 b=2 \times 10^{8}\right)$

Figure 14: Backface strain evolution in SG locations at $40 \%$ of $\tau_{S}\left(z=8 \quad b=2 \times 10^{8}\right)$

Figure 15: Experimental compared to modelling load-life data

Figure 16: Experimental compared to modelling strain for different SGs, $50 \% \tau_{S}$

Figure 17: Experimental compared to modelling strain for different SGs, $30 \% \tau_{S}$

Figure 18: Experimental compared to modelling strain for different SGs, $40 \% \tau_{S}$ 